\title{
Impact of an interest in asthma on prescribing costs in general practice
}

Kevin Jones

General Practitioners in Asthma Group
Faculty of Medicine, University of Southampton, Aldermoor Health Centre, Southampton SO1 6ST

Kevin Jones, senior lecturer in primary medical care and study coordinator Accepted for publication 28 April 1992

\begin{abstract}
Objective - To examine the effect on total prescribing costs and prescribing costs for respiratory drugs for practices with at least one general practitioner with a special interest in asthma.

Design - Postal questionnaire survey. Setting - General practitioners in England and Wales.

Subjects - 269 members of the General Practitioners in Asthma Group, of whom 103 agreed to participate.

Main measures - Individual practitioners' and their practices' PACT prescribing costs from the winter quarters of 1989-90 compared with average costs for their family health services authority (FHSA) and a notional national average of all FHSAs combined.

Results - The response rate was $57 \%$; the average total prescribing costs for the practices of the 59 respondents were significantly lower than those of their respective FHSAs (mean difference $\mathbf{t 5 0 5}$ per 1000 patients per quarter $(95 \%$ confidence interval $-934 \cdot 0$ to $-76 \cdot 2$, $p=0.022$ ) and lower than the national average. The average prescribing costs for respiratory drugs for the practices were significantly greater than those for their FHSA ( $£ 195$ per 1000 patients per quarter $(84.4$ to $306 \cdot 0, p=0.001)$ and the national average. Both types of costs varied widely.
\end{abstract}

Conclusion - An interest in asthma care in general practice is associated with higher average prescribing costs for respiratory drugs but no increase in overall prescribing costs compared with those for respective FHSAs and national averages.

Implications - FHSAs and their medical advisors should not examine high prescribing costs for individual doctors or one therapeutic category but in the context of practice total costs.

(Quality in Health Care 1992;1:110-113)

\section{Introduction}

The introduction of indicative prescribing budgets and the role of the new medical advisors to family health services authorities (FHSAs) in monitoring prescribing costs suggest that prescribing analyses and cost (PACT) data will become much more important. PACT data comprise a quarterly report on prescribing costs sent to all general practitioners in England and Wales from the Prescription Pricing Authority in Newcastle upon Tyne. Level 1 data give prescribing costs under several summary headings, but level 2 and 3 data, which are available on request, give more details concerning the individual drugs and preparations prescribed. Practices with high costs, either in total or in any of the subgroups based on the British National Formulary, may have to justify to the FHSA their apparent excess in drug expenditure.

At the same time general practitioners are being exhorted to make greater use of antiinflammatory drugs in treating asthma in adults $^{1}$ and children $^{23}$ to suppress the inflammatory changes found in the bronchi of even mild asthmatic patients, ${ }^{4}$ to reduce the mortality and morbidity from asthma that has been called for in community care, ${ }^{5-7}$ and thus to improve the quality of care delivered to patients with asthma. However, these antiinflammatory medicines are expensive, and increases in their use will certainly raise prescribing costs for patients with respiratory conditions. What is not clear, however, is what influence such changes will have on overall prescribing costs.

This paper reports a study of total prescribing costs and prescribing costs for respiratory drugs for general practitioners with a special interest in asthma (members of the General Practitioners in Asthma Group) and their practices and compares them with the average prescribing costs for their FHSA. The current prescribing costs for respiratory drugs for members of the group are predicted to reflect future trends in national prescribing costs if increased prescribing of antiinflammatory drugs does occur.

\section{Subjects and methods}

All 269 members of the General Practitioner in Asthma Group based in England and Wales received a single postal invitation to participate in the survey. One hundred and three members agreed to participate and were sent a questionnaire which asked for the following information: name of FHSA; list size (as stated on the PACT return); number of partners in the practice and their hours (26, 19 , or 13); total prescribing costs; prescribing costs for respiratory drugs (including prophylactics) and for prophylactic respiratory drugs alone for the individual practitioner, the practice as a whole, and the average cost for 
the FHSA (not available for prophylactic drugs); and the quarter end date (the last available quarter of PACT data for 1989 was to be used). The quarter end date was needed because PACT data are supplied to general practitioners with differing dates, depending on geographical factors. The cost of prophylactic drugs was derived by recording the sums of sections $3 \cdot 2$ (inhaled corticosteroids) and 3.3 (sodium cromoglycate, nedocromil sodium, and ketotifen) of the PACT data. The questionnaire was accompanied by supporting notes explaining how to obtain the required level 1, 2, and 3 PACT reports. Nonresponders received a repeat questionnaire after about three weeks. It should be noted that prescribing costs for respiratory drugs also include those for drugs for allergic disorders (mainly antihistamines), respiratory stimulants, oxygen, mucolytics, aromatic inhalations, antitussives, and systemic nasal decongestants.

The total number of partners and their time commitments were used to compute a notional share of the list as follows: the sum of one times the number of 26 hour principals, 0.75 times the number of 19 hour principals, and 0.5 times the number of 13 hour principals was divided into the practice list size to give a notional share of the list size. If responders among this group of general practitioners had a time commitment below 26 hours, the share was reduced by either a factor of 0.75 or 0.5 as appropriate. Individual, practice, and average FHSA costs could then be expressed as cost per 1000 patients and these figures compared between practices of differing sizes.

Comparisons could then be made for total prescribing costs and prescribing costs for respiratory drugs between the individual practitioner and the practice as a whole, the individual practitioner and the FHSA, the practice as a whole and the FHSA, and the practice and a notional national average computed as the mean of all the FHSA averages supplied. Costs of prophylactic drugs could be compared between only the individual practitioners and their practices.

Data were analysed with the SPSS-PC+ package for IBM personal computers ${ }^{8}$ and the Confidence Interval Analysis package of Gardner et al. ${ }^{9}$ Comparisons between groups were made with paired two tailed $t$ tests.

\section{Results}

Replies were obtained from 59 members of the General Practitioners in Asthma Group, representing a response rate of $57 \%$ of the 103 general practitioners who agreed to participate and $22 \%$ of the total number of members of the group in England and Wales. Not all replies were complete as full PACT data were not available to all respondents in the timescale of the study. The data came from quarters ending between October 1989 and February 1990 and thus represent a mixture of autumn and winter prescribing. Forty one FHSAs out of the total 92 in England and Wales were included in the responses, and
Table 1 Prescribing costs ( $\_$per 1000 patients per quarter) for individual practitioners, practices, and FHSAs

\begin{tabular}{lccc}
\hline & $\begin{array}{c}\text { Individual } \\
\text { participating } \\
\text { practitioners }\end{array}$ & $\begin{array}{c}\text { All practitioners } \\
\text { in participating } \\
\text { practices }\end{array}$ & $\begin{array}{c}\text { All practitioners } \\
\text { in participating } \\
\text { FHSAs }\end{array}$ \\
\hline All drugs: & & & \\
No & 54 & 57 & 39 \\
Mean & 10151 & 9555 & 10220 \\
Range & $1209-18311$ & $2500-16189$ & $7302-13089$ \\
SD & 3827 & 2298 & 1568 \\
Respiratory drugs: & 50 & 57 & 39 \\
No & 1764 & 1271 & 1080 \\
Mean & $244-5713$ & $175-2967$ & $184-1497$ \\
Range & 1083 & 470 & 240 \\
SD & & & \\
Prophylactic respiratory drugs: & 37 & \\
No & 50 & 685 & \\
Mean & 955 & $48-1510$ & \\
Range & $53-3222$ & 370 & \\
SD & 697 & & \\
\hline
\end{tabular}

practices ranged from 1 to 10 (median $4 \cdot 75$ ) partners. List sizes varied from 2316 to 22836 (mean 9014 (SD 4505)) and individual shares of the list size varied from 1062 to 3110 (mean 2054 (SD 491)).

Table 1 shows the mean total prescribing costs and the prescribing costs for respiratory and prophylactic drugs per 1000 patients per quarter for the individual members of the group, for the practice as a whole, and for the FHSAs, when all available data are used. In the following paired comparisons, where more complete data were present, all figures are expressed as cost in pounds per 1000 patients per quarter. The total prescribing costs for individual practitioners were not significantly different from the average total costs for the practice $(n=54$, mean difference $£ 594$ (95\% confidence interval $-£ 256$ to $£ 1444, t=1 \cdot 4$; $p=0 \cdot 166)$ or from the average total costs of the corresponding FHSA $(\mathrm{n}=54$, mean difference $£ 39$ (95\% confidence interval $-£ 934$ to $£ 1012, t=0.08 ; \mathrm{p}=0.936)$. The average total costs for the practices were significantly less than those of the corresponding FHSAs $(n=56$, mean difference $-£ 505$ (95\% confidence interval $-£ 934.0$ to $-£ 76.2, t=-2 \cdot 36 ; \mathrm{p}=0.022)$.

The prescribing costs for respiratory drugs for the participating general practitioners were greater than the average total costs for their practices $(n=50$, mean difference $£ 459$ (95\% confidence interval $£ 242$ to $£ 676, t=4 \cdot 26$; $\mathrm{p}<0.001)$ and greater than those for their FHSA ( $n=49$, mean difference $£ 676$ (95\% confidence interval $£ 371$ to $£ 981, t=4.46$; $\mathrm{p}<0.001)$. Similarly, the practices' average prescribing costs for respiratory drugs were greater than those for their FHSA $(n=56$, mean difference $£ 195 \quad(95 \%$ confidence interval $£ 84.4$ to $£ 306.0, \quad t=3.53$; $\mathrm{p}=0.001)$. Costs of prophylactic drugs could be compared between only the individual practitioners and their practices in a smaller number of cases; the average prophylactic costs for individual practitioners were greater than those for the practice $(n=34$, mean difference $£ 371$ (95\% confidence interval $£ 188$ to $£ 554, t=4 \cdot 12 ; \mathrm{p}<0.001)$.

Table 2 shows these comparisons expressed as percentages and also includes the relation of individual and practice average costs to the national average. 
Table 2 Relations between average prescribing costs for individual participating practitioners, practices, FHSAs, and national average

\begin{tabular}{|c|c|c|}
\hline Cost & Mean \% & $95 \%$ Confidence interval \\
\hline \multicolumn{3}{|c|}{ Individual practitioner versus practice } \\
\hline Total drugs & 106 & 98 to 115 \\
\hline Respiratory drugs & 129 & 115 to 143 \\
\hline Prophylactic drugs & 146 & 127 to 166 \\
\hline \multicolumn{3}{|c|}{ Individual practitioner versus $F H S A$} \\
\hline Total drugs & 100 & 91 to 110 \\
\hline Respiratory drugs & 162 & 134 to 189 \\
\hline \multicolumn{3}{|c|}{ Practice versus FHSA } \\
\hline Total drugs & 95 & 90 to 99 \\
\hline Respiratory drugs & 118 & 108 to 128 \\
\hline \multicolumn{3}{|c|}{ Practice versus national average (mean of FHSA averages) } \\
\hline Respiratory drugs & 118 & 106 to 130 \\
\hline
\end{tabular}

\section{Discussion}

Ever since Speight $e t$ al showed that morbidity among schoolchildren with asthma could be reduced by prescribing anti-inflammatory drugs, ${ }^{10}$ there has been much confirmatory evidence that increased use of such drugs leads to a better quality of care. That evidence has been encapsulated in the series of consensus statements about management of asthma which have emerged on both sides of the Atlantic. ${ }^{1-3} 1112$ PACT costs may indicate general practitioners' prescribing behaviour as the anti-inflammatory drugs are much more expensive than respiratory drugs, but they cannot be directly related to quality of care, as they do not necessarily reflect the utilisation of anti-inflammatory drugs nor their influence on morbidity or their effect, if any, on use of medical resources. The number of asthmatic patients treated are also not accessible from PACT data.

The recently introduced concept of indicative prescribing budgets ${ }^{13} 14$ makes it essential to examine the possible effects on prescribing costs of the recent recommended changes in asthma management. ${ }^{1-3} 1112$ Underdiagnosis, delayed diagnosis, and undertreatment of asthma are commonplace in the community, especially in children, ${ }^{10}{ }^{15-17}$ and our highly selected sample of practices which included a partner with a special interest in asthma enabled potential increases in average prescribing costs of respiratory drugs to be studied.

Our study had several drawbacks. The response rate to our survey was low, primarily because of the limited time available for data collection, and a larger sample might have included less committed practices. However, the results are still worth considering because they are likely to reflect the prescribing practice of practitioners most involved in improving asthma care. Membership of the General Practitioners in Asthma Group does not entail any subscription, and thus many of the non-respondents may not be trying particularly to improve asthma care in their practices and their prescribing costs would be likely to be near the means. In addition, prescribing costs for respiratory drugs include drugs which are not normally part of asthma treatment. Including only those drugs in sections $3 \cdot 1$ (bronchodilators), $3 \cdot 2$ (corticosteroids), and $3 \cdot 3$ (prophylaxis of asthma) of the British National Formulary would have been preferable, but FHSA averages for these headings would have been necessary, and these are not available from the PACT information sent to practices. Data on prescribing costs for antibiotics might have been helpful as they might have shown considerable cost savings, but we did not seek this information. Conversion of FHSA averages from the figures given by the practices to cost per 1000 patients may contain potential errors. In the PACT reports FHSA averages are calculated on the basis of the number of "prescribing units" in the practice, not the number of patients. Each patient on the lists who is older than 65 years is regarded as three prescribing units, and differences in patients' age profiles among practices may have influenced our findings. The FHSA average figures should properly be regarded as costs per quarter per 1000 patients of similar age profile.

Despite these potential sources of error our results provide some useful data which suggest that high prescribing costs in asthma prophylaxis and respiratory drugs overall may be associated with savings in other areas of care. The practices surveyed had average total prescribing costs which were less than those of both their own FHSA and FHSAs combined. The reasons for this are open to speculation, but one possible factor must be that practice teams including a member with an interest in asthma are more likely to be among those trying to deliver better care in general and rational prescribing in particular. It is also clear, however, that the prescribing costs for respiratory drugs for this sample were significantly higher than those for their FHSA and FHSAs combined.

Individual members of the group on average had higher prescribing costs for respiratory drugs than their practices, probably reflecting the disproportionately higher number of asthmatic patients they see and prescribe for. However, the data on individuals' prescribing costs must be interpreted with caution. The concept of the notional share of the list described is an attempt to compare between individual practitioners, but it may well not reflect responsibility for patient care or prescribing. Moreover, PACT data do not identify repeat prescriptions nor clinical responsibility by individual doctors. The PACT data we analysed might have included large numbers of prescriptions written by the partners of the doctors surveyed, as we did not ask about the existence of personal lists or protocols for use of prescription pads. Nevertheless, the fact that the practices' overall prescribing costs for respiratory drugs were higher than the average for their FHSA suggests that index general practitioners were not simply "cornering the practice market."

The government's working paper on indicative prescribing budgets in 1989 made clear the wide variations in prescribing between FHSAs - from $£ 26000$ to $£ 40000$ per 1000 patients in $1986-7 .{ }^{14}$ Our results reinforce this for total prescribing costs and 
prescribing costs for respiratory drugs for the practices and FHSAs in our sample. Total prescribing costs for FHSAs in our survey varied from $£ 7302$ to $£ 13441$ per 1000 patients per quarter and prescribing costs for respiratory drugs from $£ 184$ to $£ 1557$. This wide variation cannot simply be attributed to variations in the population and must be influenced by doctors' behaviour in relation to prescribing, investigations, and referral which are known to vary widely. Investigating this variation is beset with difficulties. ${ }^{18}$ For example, one study of general practitioners with high and low referral rates not only failed to elucidate appreciable differences in the doctors or their practices but also showed no consistent link with prescribing or use of investigations. ${ }^{19}$

The estimation of indicative budgets on the basis of FHSA averages may be rather misleading if prescribing is to be made more rational as well as cheaper, particularly in specific areas such as management of asthma. Indeed, applying national averages to prescribing for asthma may also be misleading as undertreatment is so widespread. FHSAs should examine high prescribing costs for respiratory drugs incurred by individual general practitioners and practices with great caution, taking into account if possible the asthma service they provide. If FHSAs were to target high costs in individual prescribing groups as potentially undesirable these practices might be subject to unnecessary and inappropriate investigation.

In conclusion, we showed that individual general practitioners with an interest in asthma have on average higher prescribing costs for respiratory and prophylactic drugs than those of their practice. Much more importantly, their practices have on average higher prescribing costs for respiratory drugs per 1000 patients than their FHSAs but lower total costs. High prescribing costs must not be examined for individual doctors, nor for one therapeutic category in isolation, but should be seen in the context of total practice prescribing costs, and FHSAs and their medical advisors need to be aware of this important concept. High prescribing costs for respiratory drugs may be an indicator of good prescribing for asthma and low costs an indicator of bad prescribing, but an essential next step is to relate differences in costs to differences in practices' prevalence of diagnosed asthma as a percentage of list size, hospital admission rates and outpatient referrals, need for rescue treatments such as oral steroids and acute nebulisations, and time missed from work or school.

The General Practitioners in Asthma Group thank Merrilyn Dracass for her help with the data handling in this study, Professor John Bain and Dr Ann-Louise Kinmonth for their advice, and our secretariat at Vox Prism for dispatching the questionnaires. This work was supported by a grant from Allen and Hanburys.

1 British Thoracic Society. Guidelines for management of asthma in adults. I. Chronic persistent asthma. $B M 9$ 1990;301:651-3.

2 Warner JO, Gotz M, Landau LI, Levison H, Milner AD, Pedersen S, et al. Management of asthma: a consensus statement. Arch Dis Child 1989;64:1065-79.

3 Asthma: a follow-up statement from an international paediatric asthma consensus group. Arch Dis Child 1992;67:240-8.

4 Beasley R, Roche WR, Roberts JA, Holgate ST. Cellular events in the bronchi in mild asthma and after provocation. Am Rev Respir Dis 1989;139:806-17.

5 Jones KP. Asthma - still a challenge for general practice. $\mathcal{F} R$ Coll Gen Pract 1989;39:254-6.

6 Management of asthma in the community. Lancet 1989;ii:199-200.

7 Jones KP. Asthma care in general practice - time for revolution? Br $\mathcal{F}$ Gen Pract 1991;41:224-6.

8 Norusis MJ. The SPSS guide to data analysis for SPSS/PC+ Chicago: Statistical Package for the Social Sciences, 1989.

9 Gardner MJ, Gardner SB, Winter PD. Confidence Interval Analysis (CIA) Microcomputer Program Manual, Version 1.0. London: $B M 7$, 1989:1-77.

10 Speight ANP, Lee DA, Hey EN. Underdiagnosis and undertreatment of asthma in childhood. BMF 1983;286:1253-7.

11 Hargreave FE, Dolovich J, Newhouse MT. The assessment and treatment of asthma: a conference report. 7 Allergy and treatment of asthma: a confer.

12 Sheffer AL. Guidelines for the diagnosis and management of asthma. National Heart, Lung, and Blood Institute national asthma education programme expert pane report. $\mathcal{f}$ Allergy Clin Immunol 1991;88:425-534.

13 Secretaries of State for Health, Wales, Northern Ireland, and Scotland. Working for patients. London: HMSO, 1989. (Cmnd 555.)

14 Indicative prescribing budgets for general medical practitioners. Working paper 4. London: HMSO, 1989.

15 Speight ANP. Is childhood asthma being underdiagnosed and undertreated? BMF 1978;ii:331-2.

16 Levy M, Bell L. General practice audit of asthma in childhood. BMF 1984;289:1115-6.

17 Kaptein AA, Dekker FW, Gill K, van der Waart MAC. Undertreatment of asthma in Dutch general practice. Undertreatment of asthma

18 Roland $M$. General practitioner referral rates. $B M \mathcal{F}$ 1988;297:437-8

19 Wilkin D, Smith AG. Variation in general practitioners' referral rates to consultants. $f R$ Coll Gen Pract $1987 ; 37: 350-2$ 